\title{
Germanium "hexa" detector: production and testing
}

\author{
Milija Sarajlić,,${ }^{\mathrm{a}, ~ *}$ David Pennicard, ${ }^{\mathrm{a}}$ Sergej Smoljanin, ${ }^{\mathrm{a}}$ Helmut Hirsemann, ${ }^{\mathrm{a}}$ \\ Bernd Struth, ${ }^{\mathrm{a}}$ Thomas Fritzsch, ${ }^{\mathrm{b}}$ Mario Rothermund, ${ }^{\mathrm{b}}$ Milan Zuvic, \\ Marie Odile Lampert, ${ }^{\mathrm{c}}$ Marzhan Askar ${ }^{\mathrm{d}}$ and Heinz Graafsma ${ }^{\mathrm{a}, \mathrm{e}}$ \\ ${ }^{a}$ Deutsches Elektronen-Synchrotron (DESY), Center for Free Electron Laser Science (CFEL), \\ Photon-Science, Detector Group, Notkestrasse 85, 22607 Hamburg, Germany \\ ${ }^{b}$ Fraunhofer Institute for Reliability and Microintegration, IZM \\ Gustav-Meyer-Allee 25, 13355 Berlin, Germany \\ ${ }^{c}$ MIRION TECHNOLOGIES (Canberra) , 1 chemin de la roseraie, 67382 Lingolsheim, France \\ ${ }^{d}$ Al-Farabi Kazakh National University, \\ 71 al-Farabi Ave., Almaty, Republic of Kazakhstan \\ ${ }^{e}$ Mid Sweden University, Universitetsbacken 1, 87131 Härnösand, Sweden \\ E-mail: milija.sarajlic@desy.de
}

ABSTRACT: Here we present new result on the testing of a Germanium sensor for X-ray radiation. The system is made of $3 \times 2$ Medipix $3 R X$ chips, bump-bonded to a monolithic sensor, and is called "hexa". Its dimensions are $45 \times 30 \mathrm{~mm}^{2}$ and the sensor thickness was $1.5 \mathrm{~mm}$. The total number of the pixels is 393216 in the matrix $768 \times 512$ with pixel pitch $55 \mu \mathrm{m}$. Medipix3RX read-out chip provides photon counting read-out with single photon sensitivity. The sensor is cooled to $-126^{\circ} \mathrm{C}$ and noise levels together with flat field response are measured. For $-200 \mathrm{~V}$ polarization bias, leakage current was $4.4 \mathrm{~mA}\left(3.2 \mu \mathrm{A} / \mathrm{mm}^{2}\right)$. Due to higher leakage around $2.5 \%$ of all pixels stay non-responsive. More than $99 \%$ of all pixels are bump bonded correctly. In this paper we present the experimental set-up, threshold equalization procedure, image acquisition and the technique for bump bond quality estimate.

KEYWORDS: Solid state detectors; X-ray detectors; Materials for solid-state detectors; Hybrid detectors.

\footnotetext{
${ }^{*}$ Corresponding author.
} 


\section{Contents}

1. Introduction $\quad 1$

1.1 Germanium properties 1

2. Technology 2

$\begin{array}{ll}2.1 \text { Sensor structure } & 2\end{array}$

2.2 Bump bonding $\quad 3$

3. Experimental set-up and results 3

$\begin{array}{ll}3.1 \text { Cooling } & 3\end{array}$

3.2 Leakage current $\quad 4$

3.3 Threshold equalization $\quad 4$

$\begin{array}{ll}3.4 \text { Background radiation } & 5\end{array}$

$\begin{array}{lc}3.5 \text { Flat field response } & 6\end{array}$

$\begin{array}{ll}3.6 \text { Estimate of the bump bond quality } & 6\end{array}$

$\begin{array}{ll}\text { 4. Conclusion } & 7\end{array}$

\section{Introduction}

For a number of experiments related to diffraction measurements, solid state physics, laser science and particle and nuclear physics it is important to have a detector which can efficiently detect X-ray energies above $20 \mathrm{keV}[1,2]$. In addition, time of response, spatial and energy resolution play a significant role. These prerequisites can be fulfilled by the use of a Germanium (Ge) sensor with Medipix3RX [4] read-out chip as a hybrid detector assembly. In 2012 we developed a single chip Ge detector with Medipix3RX read-out [3,4]. Since then, we developed and tested a Ge "hexa" detector, consisting of a monolithic Ge sensor hybridized to six Medipix3RX read-out chips.

There are other so called "high-Z" materials which also have high efficiency for X-rays above $20 \mathrm{keV}$. Examples of these materials are GaAs and CdTe. GaAs gives uniform distribution in response to the X-ray flat field but with pronounced "island-like" structures $[5,6,7]$. CdTe has more narrow distribution than GaAs in response to the flat field but it shows pronounced "vein-like" structures [5,6,7]. Ge gives uniform response to the X-ray flat field, comparable to that from GaAs without any visible additional structures [3].

\subsection{Ge properties}

Ge has an atomic weight 72.6 a.u. and mass density $5.32 \mathrm{~g} / \mathrm{cm}^{3}$ at $300 \mathrm{~K}$ [8]. Its energy gap is $0.66 \mathrm{eV}$ and its intrinsic carrier density is $2.4 \times 10^{13} \mathrm{~cm}^{-3}$ at $300 \mathrm{~K}$. This is three orders of magnitude more than Silicon (Si) at the same temperature, $1.5 \times 10^{10} \mathrm{~cm}^{-3}$. This also makes a huge difference in intrinsic resistivity between $\mathrm{Si}$ and $\mathrm{Ge}$ at room temperature, with 


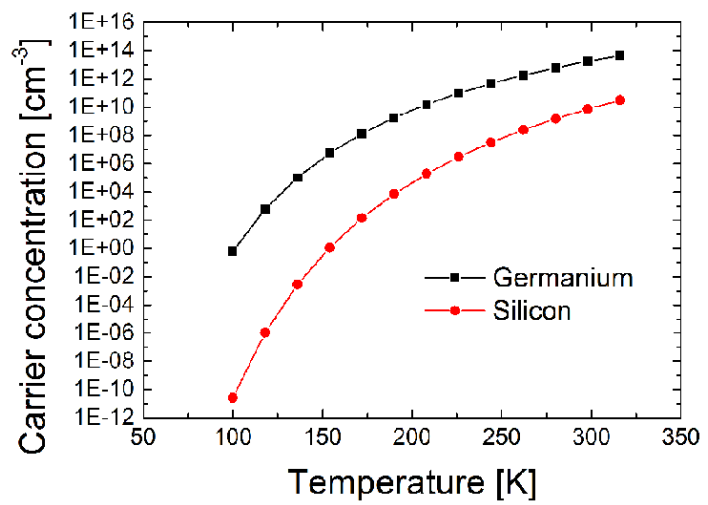

Figure 1. Intrinsic carrier density for $\mathrm{Si}$ and $\mathrm{Ge}$ depending on the Temperature.

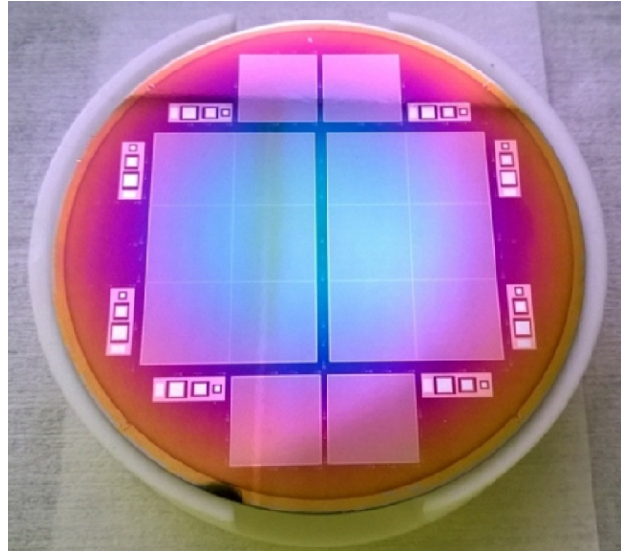

Figure 2. Ge wafer with two hexa sensors and four single sensors. Visible are also test diodes closer to the rim of the wafer. Diameter of the wafer is $90 \mathrm{~mm}$ with the thickness $1.5 \mathrm{~mm}$.

$2.3 \times 10^{5} \Omega \mathrm{cm}$ for Si vs. $47 \Omega \mathrm{cm}$ for Ge at $300 \mathrm{~K}$. When a semiconductor is used as a sensor material for X-ray detection, its leakage current plays a key role, as high leakage current will increase the detector noise and possibly saturate the read-out electronics. In order to reduce leakage and make Ge useful for X-ray detection it has to be cooled before operation to the temperatures where leakage becomes comparable to that of Si. Comparing intrinsic carrier density at lower temperatures, Figure 1, we conclude that somewhere around $200 \mathrm{~K}$ or $-80^{\circ} \mathrm{C}$ Ge sensor should be applicable for X-ray detection. This is the main reason why Ge based X-ray detectors need to be cooled before operation. Main advantage of Ge in comparison with $\mathrm{Si}$ is its higher efficiency for X-ray detection. For example, at $20 \mathrm{keV}$ X-ray energy, mass attenuation coefficient for Ge is $42.22 \mathrm{~cm}^{2} / \mathrm{g}$ vs. $4.46 \mathrm{~cm}^{2} / \mathrm{g}$ for $\mathrm{Si}$ [9]. Converted to absorption length this is $2.3 \times 10^{-2} \mathrm{~cm}$ for Ge and $2.2 \times 10^{-1} \mathrm{~cm}$ for Si. The Ge is showing 10 times shorter absorption length than $\mathrm{Si}$ at $20 \mathrm{keV} \mathrm{X}$-ray energy.

\section{Technology}

\subsection{Sensor structure}

The sensor was fabricated by Canberra Company, France [10]. "Hexa" relates to the specific structure of the sensor, which is made in the form of $3 \times 2$ Medipix3RX chips. Medipix $3 R X$ itself has a structure of $256 \times 256$ pixels which form a square matrix with a pitch of $55 \mu \mathrm{m}$. The Ge hexa is compatible to Medipix3RX in the sense of pixel matrix pitch and size. The Ge hexa's active area is $45 \times 30 \mathrm{~mm}^{2}$ and the sensor thickness $1.5 \mathrm{~mm}$. This relatively large thickness was chosen to improve mechanical stability. Another reason for the large thickness is that it would have made it possible to reprocess the wafer if there were errors during fabrication. The total number of the pixels is 393216 in the matrix $768 \times 512$ with pixel pitch $55 \mu \mathrm{m}$. The structure of the hexa sensor is such that in both directions, $x$ and y, after 255 normal pixels with pixel pitch of $55 \mu \mathrm{m}$ comes two pixels with three times larger width or height depending in which direction we are looking at. Figure 2 shows one of the Ge wafers with two hexa sensors and four single sensors. The wafer size was $90 \mathrm{~mm}$ diameter with $1.5 \mathrm{~mm}$ thickness. In total, two wafers were processed, one of which has been used for the module's assembly. 


\subsection{Bump bonding}

Bump bonding was performed by Fraunhofer IZM Institute, Berlin, Germany [11]. Special attention was paid that during bonding the Ge sensor is never heated to the temperatures above $80^{\circ} \mathrm{C}$. Temperatures higher than $80^{\circ} \mathrm{C}$ can disturb the structure of Ge material and deteriorate electrical properties by allowing impurities to diffuse deeper into material. In the process of bump bonding every pixel of the read-out chip is mechanically and electrically connected to the corresponding pixel of the sensor by a metallic bump. This process is done in a reflow oven where the whole assembly is gradually heated to the specific temperature needed to melt solder paste and form bump bond between the pixels. We opted for pure Indium as a solder material. Indium has a melting point at $156.6^{\circ} \mathrm{C}$ and due to its softness it is possible to do bonding by thermosonic compression at $80^{\circ} \mathrm{C}$, where a combination of moderate temperature, pressure and sonic energy are used to form the bump. Indium remains ductile at low temperature which reduces thermal stress on the bumps during cooling.

\section{Experimental set-up and results}

Figure 3 (left) shows the schematic of Ge system with a single chip as designed in Solide Edge software before production and assembly. Figure 3 (right) shows the Ge hexa module mounted on a LTCC ceramic circuit board [6]. Medipix3RX chips are wire bonded to the pads on the ceramics in order to make an electrical contact. Between module chips and ceramic, Indium foil is used in order to increase the heat transfer. On the other side of the ceramic there is the mechanical and thermal contact with a copper holder which in turn is connected to the cold finger of a Cryo-Tiger cooling machine. On the other side of the ceramic, a 500-pin connector is mounted which provides electrical connection to the Signal Distribution (SD) board. The SD board is placed in the vacuum barrier flange, which is sitting in the back flange of the vacuum chamber. On the other side of vacuum flange contacts for electronics, Ethernet, temperature sensors, vacuum pumps and Cryo-Tiger cooling are provided. On the front side of the vacuum chamber, a flange with a Mini-X X-ray tube produced by company Amptek [12] is fixed by clamps and a KF-type Centering-Ring Seal assembly.

\subsection{Cooling}

The system with the Ge hexa module is closed in a vacuum chamber and pumped down to approximately $2 \times 10^{-3} \mathrm{mbar}$ pressure. This is needed to prevent icing on the surface of the detector during cooling. The detector is cooled by the Cryo-Tiger cooling machine to the temperature $-126^{\circ} \mathrm{C}$, which was the lowest temperature that could be reached with this type of cooling. The temperature was measured by a Pt1000 resistor mounted on the LTCC ceramic next to the detector chips. When the detector is powered, total power consumption was $30.7 \mathrm{~W}$ while performing threshold equalization would increase total power to $38.4 \mathrm{~W}$. This total power includes also the power consumed by FPGA and read-out electronics. Every Medipix3RX read-out chip is dissipating around $1.2 \mathrm{~W}$ of power and increasing the temperature of the system. This makes an additional challenge in keeping Ge sensor at low temperatures during operation of Medipix3RX chips. After half an hour of operation of the Ge hexa detector, the temperature at the measuring point on top of the ceramic would converge to $-117^{\circ} \mathrm{C}$. This we consider as working temperature of the system. We expect that any temperature below $-100^{\circ} \mathrm{C}$ should be good enough for operation of the Ge detector. 

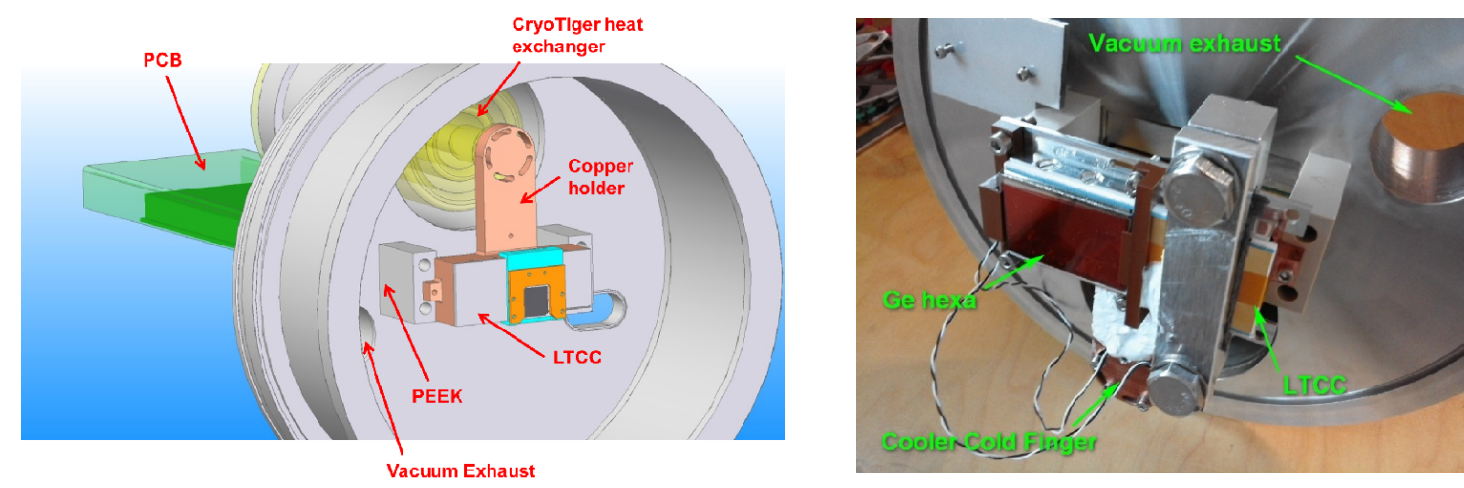

Figure 3. Left: Schematic of the Ge module with the single chip attached. Right: Ge hexa module as assembled on the back vacuum flange.
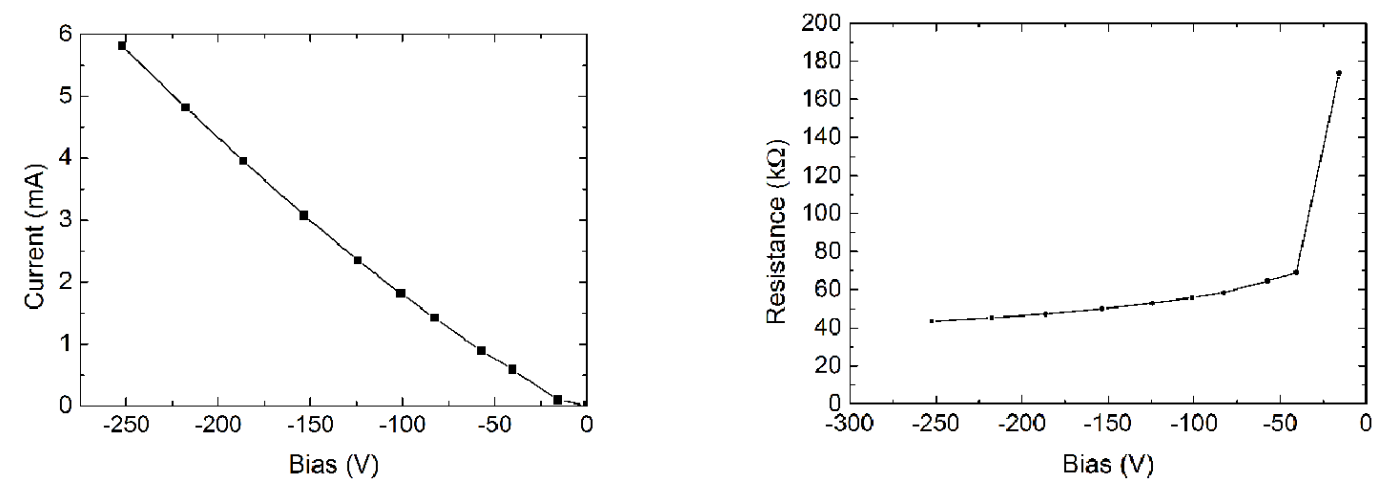

Figure 4. Current-voltage (left) and resistance-voltage (right) characteristics of the Ge hexa sensor on the temperature $-126 \mathrm{C}$.

\subsection{Leakage current}

The leakage current of the Ge hexa sensor was measured at $-126^{\circ} \mathrm{C}$ while varying the polarization bias applied on the sensor. Figure 4 (left) shows the leakage current vs. bias in the range from zero to $-300 \mathrm{~V}$. The current is almost linearly rising from zero to the values up to $6 \mathrm{~mA}$. This could be the consequence of the increased leakage on the sensor rim caused by the surface defects made by chip dicing in the process of manufacturing. Figure 4 (right) depicts the sensor resistance vs. bias. The resistance starts from relatively high values and drops rapidly to something around $40 \mathrm{k} \Omega$. There is a built in resistor in the circuitry with the resistance $20 \mathrm{k} \Omega$, which means that the resistance of the Ge sensor is converging to the value slightly above $20 \mathrm{k} \Omega$. This might induce that the Ge sensor is behaving more like a resistor then as a diode. This could be the consequence of a leakage current present on the rim of the Ge sensor which is caused by material defects on the rim which are in turn introduced during sensor dicing.

One more consequence of the leakage current is blocking responsiveness of the Medipix3RX pixels. Every pixel in the read-out chip has a current compensation circuitry which is compensating for the leakage current. If a leakage is too high, the current cannot be compensated anymore, and the pixel becomes non-responsive.

\subsection{Threshold equalization}

The so-called threshold equalization procedure is performed in order to equalize all noise edges of various pixels and make response of the detector as uniform as possible. For the sake 

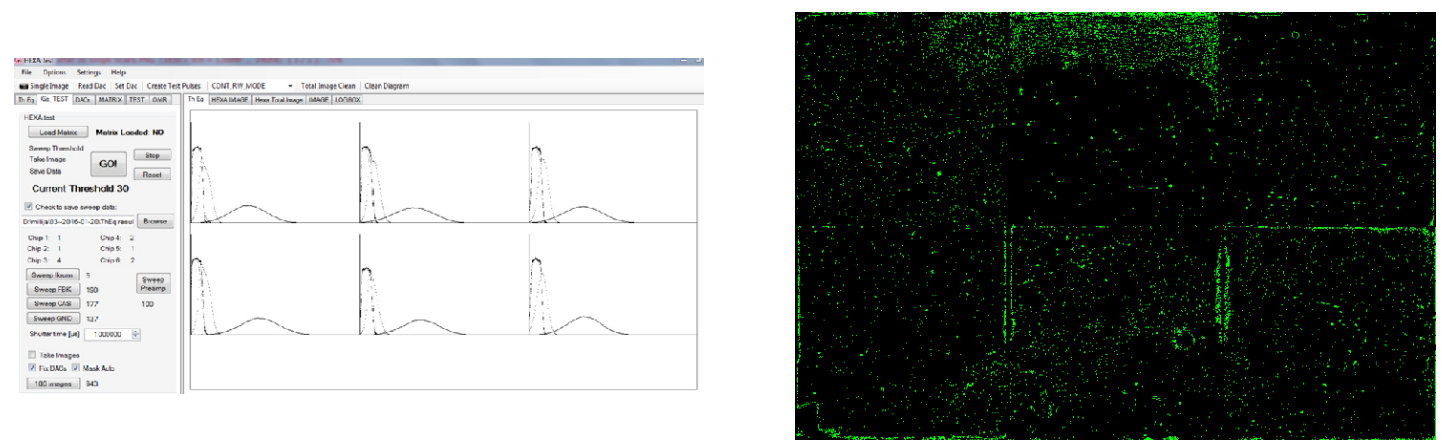

Figure 5. Left: Screenshot of GUI of the software for threshold equalization and data acquisition. Right: Image depicting masked pixels after 384 seconds of background radiation acquisition. Masked pixels are painted green, normal pixels are black.

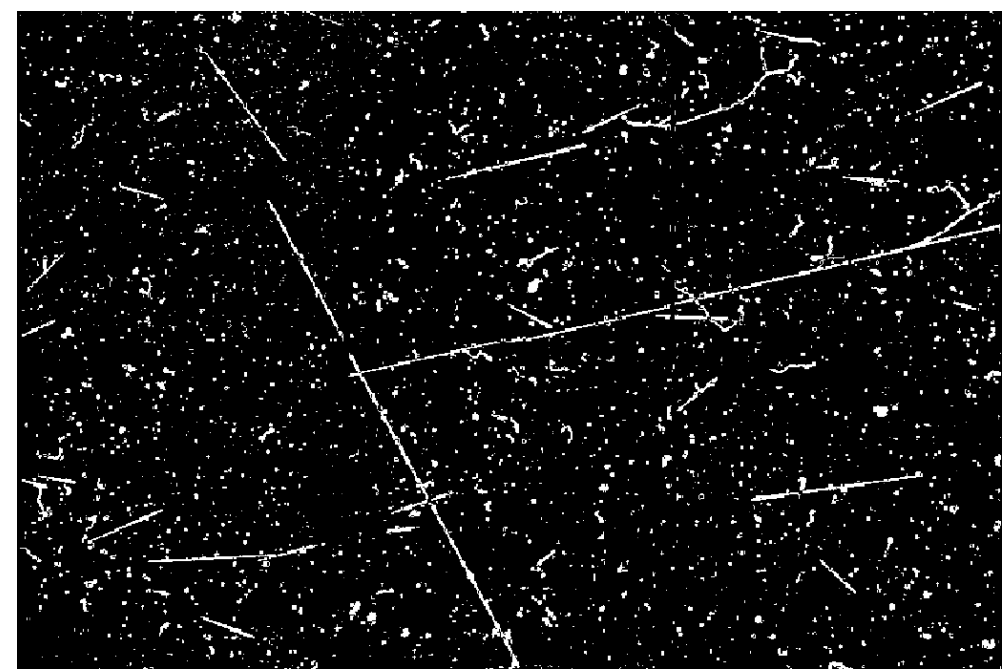

Figure 6. An example of a background radiation as recorder by Ge hexa detector. Image acquisition time was 384 seconds. Black pixels have zero count and white pixels show two counts.

of threshold equalization a novel procedure is designed and implemented in the read-out software. The aim of this novel procedure was to complete the threshold equalization in a limited time. This prerequisite was driven by the fact that threshold equalization is strongly dependent on the temperature of the read-out chip and sensor. For that reason it is favourable to complete the full process sooner in order to avoid larger temperature fluctuations. We developed a new algorithm in order to equalize Ge sensor faster. It is completing in $10 \mathrm{~min}$ and we are satisfied with this speed. The procedure works in two steps. First we scan all threshold values and collect total pixel counts per chip. We find the maximum value of the noise counts and calculate the configuration based on an approximate formula. In the second step we scan thresholds again but this time we compare noise edges in respect to the wish noise edge position and make fine tuning of the configuration matrix. Figure 5 (left) gives screenshot of the GUI of the software for threshold equalization and data acquisition.

\subsection{Background radiation}

After the threshold equalization is finished software can do automatic noisy pixel masking. In this procedure all pixels counting more than two counts are masked. We end up with around 2000 or $0.5 \%$ masked pixels. Benefit of this procedure is that after masking we end up with remaining pixels which are absolutely quiet in the sense that they produce no counts as a 
consequence of internal Medipix3RX noise or leakage coming from the sensor. The detector is now ready to sense very low activity like background radiation. Figure 6 shows an example of the background radiation as detected by Ge hexa sensor. The image is a result of the 384 seconds acquisition time. Long straight lines are typically made by heavy particles like protons, neutrons or muons while curved lines are made by light particles like electrons. Ions or alpha particles would make blobs which are visible at some parts of the image. Black pixels at Figure 6 count zero and white pixels count two. It is not clear to us at this point why every event of the background radiation is producing two counts. Normally, we would expect one count per event. It would require more investigations to determine the cause of this behaviour.

The procedure for masking the noisy pixels is running during acquisition of background radiation thus accumulating more noisy pixels. At the end of background radiation acquisition or after 384 seconds, total number of masked pixels has climbed to around 15000 or $3.7 \%$ of the total pixel number, Figure 5 (right). The masked pixels show particular pattern as presented at Figure 5 (right). The masked pixels are painted green and they appear mainly around non-responsive pixels which are blocked by the high leakage current.

\subsection{Flat field response}

The next test was the response to the flat field X-ray illumination. The flat field was produced using a Mini-X X-ray tube from company Amptek. This tube is producing X-rays in the range from 5 to $50 \mathrm{keV}$ with two clear peaks at 22 and $25 \mathrm{keV}$. Medipix $3 \mathrm{RX}$ settings were: Super High Gain, Ikrum $=5$, ThresholdLow $=30$, Shutter Time $=1$ second, High Voltage $=-200 \mathrm{~V}$, Leakage Current $=4.8 \mathrm{~mA}$. Figure 7 (left) gives the flat field image after 61 second acquisition time. The histogram distribution of the flat field image is given in figure 7 (right). In figure 7 (left) it is visible that some of the surfaces are dark and pixels are not giving counts. This is mainly happening along the rim of the sensor and along the large pixels which are positioned between Medipix3RX chips. These large pixels have also large surface in respect to the normal pixels and therefore drive more leakage current. Higher leakage around the rim of the sensor is caused by the non-optimised Guard Ring structure which forms the electrostatic field inside of the sensor. It could be also caused by surface defects in the Ge material caused by dicing of the sensor chips. In addition there are few black spots inside of the lower central, lower right and upper right chip. This could be the consequence of the defects in the Ge material which is causing higher conductivity than the rest of the sensor, thus increasing the leakage and blocking the pixels. Uniformity of the pixel response to the Flat Field is relatively good as visible on the histogram in Figure 7 (right). Histogram is made with the bin size 0.2 and is fitted with Gaussian distribution using least squares method. The mean value is calculated to be 0.62, standard deviation 0.063 and FWHM 0.148. Pixels which are not responding constitute around $2.5 \%$ of the total pixel number.

\subsection{Estimate of the bump bond quality}

Bump bond quality was estimated by a procedure specially developed for this purpose. The detector was placed in the climate chamber and cooled down to $-40^{\circ} \mathrm{C}$. We use one of the parameters which could be set inside of the Medipix3RX chip, so-called Ikrum. Figure 8 (left) shows the total number of counts for the whole sensor vs. Ikrum value for fixed ThresholdLow at 85 . It is visible that the number of total counts is very low for the low Ikrum values but it suddenly starts to grow when Ikrum goes over certain value. Our interpretation of the result is this. The Ikrum parameter is controlling current compensation circuitry and is defining amount of the leakage current which can be compensated. Low leakage is producing small number of 

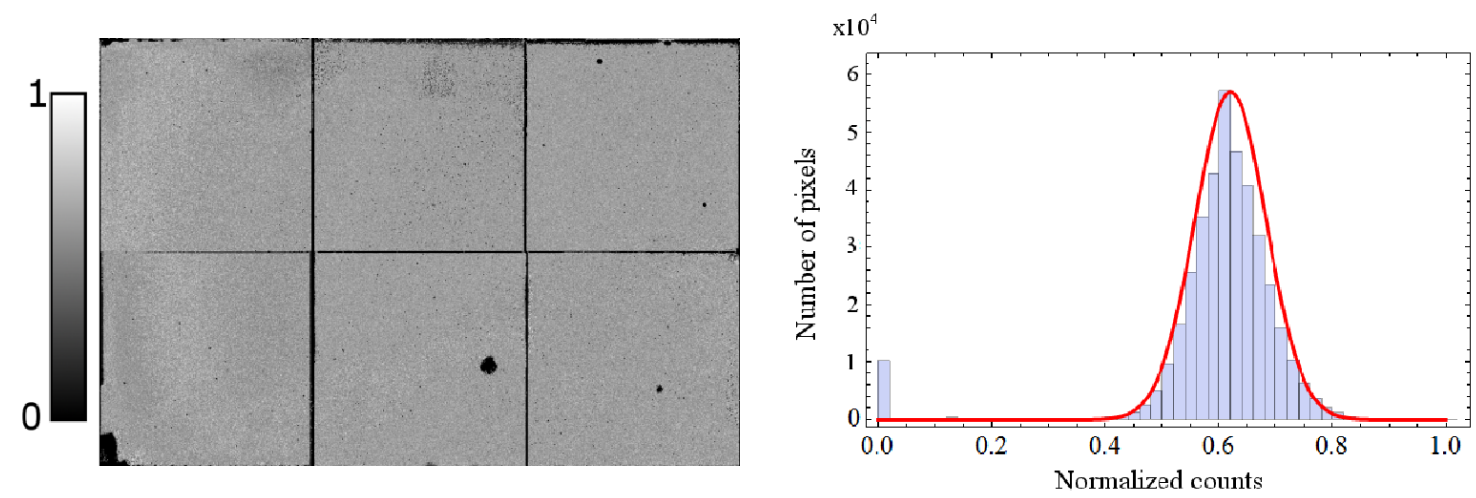

Figure 7. Left: flat field image after 61 second acquisition time. Right: Histogram of the same image (bars), Gaussian distribution fit with the mean value 0.62, standard deviation 0.063 and FWHM 0.148, (red line).
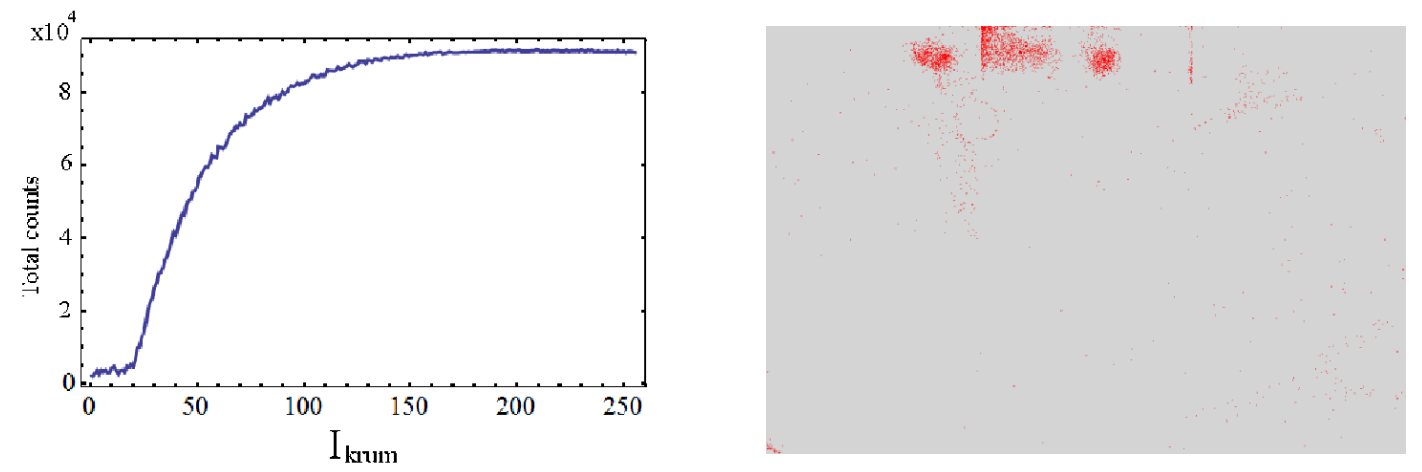

Figure 8. Left: Total number of counts vs. Ikrum value. Right: Image of estimated bump bond quality. Red pixels are not properly bonded while grey pixels are normal.

counts in pixel. More leakage is producing more. But when leakage is above certain level, pixel will stop counting. If the leakage current is already too high for the pixel to respond than higher Ikrum is suppressing leakage current influence and makes more pixels to respond. Lower Ikrum is lowering leakage that can be compensated, thus more pixels become non-responsive. At the lower limit, when Ikrum is low enough, all pixels should be blocked. If some of them still appear noisy they actually see the noise from Medipix3RX chip itself. We may assume that they are not bonded properly. We estimated 2851 pixels or $0.72 \%$ of all pixels as non-bonded. Depiction of the non-bonded pixels estimated in this way is given in Figure 8 (right). Comparing Figure 8 (right) and flat field image in Figure 7 (left) it is visible that upper left chip in the upper right corner shows darker region which could be attributed to the non-bonded pixels. Also upper part of the upper central chip is affected by the presence of non-bonded pixels. Part of the signal in flat field image comes from the intrinsic noise in Medipix3RX chip that is why non-bonded pixels look only darker and not totally black. There are no masked pixels in this procedure. This procedure is applicable only to Ge sensor because of the high leakage current which it has.

\section{Conclusion}

We have fabricated and tested a Ge based pixelated hybrid X-ray detector. This is a novel design with six times increased surface of the sensor in respect to the previous system. This detector could be equalized and configured to detect low intensity signals like background 
radiation with great accuracy. It also has high responsiveness to the $\mathrm{X}$-radiation produced by $\mathrm{X}$-ray source thus giving relatively good uniformity across the matrix of the sensor. We also developed the procedure for estimate of the bump bond quality and our prediction gives a good match with the sensor response on the flat field.

We plan to use this sensor as the basis for even larger Ge pixelated detector which should consist out of the matrix of $3 \times 2$ Ge hexa detectors.

\section{Acknowledgments}

The authors wish to thank to the Medipix3RX designers Rafael Ballabriga Sune and Xavi Llopart Cudie, Jerome Alexandre Alozy and CERN Microelectronics Group leader Michael Campbell for their help on the details of Medipix3RX technology and functionality.

\section{References}

[1] S. Ehn et al., X-ray deconvolution microscopy, Biomedical Optics Express 7 (2016) 4 doi: 10.1364/BOE.7.001227.

[2] F. Westermeier et al., Connecting structure, dynamics and viscosity in sheared soft colloidal liquids: a medley of anisotropic fluctuations, Soft Matter 12 (2016) 171 doi: 10.1039/c5sm01707f.

[3] D. Pennicard et al., A germanium hybrid pixel detector with 55 $\mu$ m pixel size and 65,000 channels, JINST 9 (2014) P12003 doi:10.1088/1748-0221/9/12/P12003.

[4] R. Ballabriga et al., The Medipix3RX: a high resolution, zero dead-time pixel detector readout chip allowing spectroscopic imaging, JINST 8 C02016, (2013). doi:10.1088/1748-0221/8/02/C02016

[5] D. Pennicard and H. Graafsma, Simulated performance of high-Z detectors with Medipix 3 readout, 2011 JINST 6 P06007 doi : 10.1088/1748-0221/6/06/P06007.

[6] D. Pennicard et al., The LAMBDA photon-counting pixel detector, J. Phys. Conf. Ser. 425 (2013) 062010 doi: $10.1088 / 1742-6596 / 425 / 6 / 062010$.

[7] D. Pennicard et al., Development of LAMBDA: Large Area Medipix-Based Detector Array, 2011 JINST 6 C11009 doi : 10.1088/1748-0221/6/11/C11009.

[8] G. Bertolini an A. Coche (eds.), Semiconductor Detectors, Elsevier-North Holland, Amsterdam, 1968.

[9] NIST, X-Ray Mass Attenuation Coefficients, https://www.nist.gov/pml/x-ray-mass-attenuationcoefficients

[10] MIRION TECHNOLOGIES (Canberra), http://www.canberra.com/default.asp

[11] Fraunhofer Institute for Reliability and Microintegration IZM, http://www.izm.fraunhofer.de/en.html

[12] AMPTEK, http://amptek.com/ 